\title{
Advances in the Clinical Diagnosis of TB in Children
}

$\mathrm{T}$ he tuberculosis (TB) epidemic remains one of the most daunting public health challenges of the $21^{\text {st }}$ century. Fuelled mainly by poverty, global population growth, the human immunodeficiency virus (HIV) epidemic and the emergence of drug resistant TB, more people suffer and die from TB each year than ever before. The TB, disease burden is carried almost exclusively by the developing world. Children in TB-endemic countries suffer severe TB-related morbidity and mortality, but this is rarely appreciated due to diagnostic difficulties and poor data collection (1). Children were previously neglected as TB control efforts focused almost exclusively on the diagnosis and treatment of adults with sputum smear-positive TB. However, the formulation of the millennium developmental goals and other recent developments raised awareness about the preventable TB disease burden suffered by children in developing countries. In response the World Health Organization (WHO) produced a guidance document on Child TB in 2006 and the Global Drug Facility (GDF), for the first time, made child friendly TB drug formulations available to poor countries in 2007.

Increased awareness and drug availability renewed the focus on the diagnostic difficulties experienced in resourcelimited settings. Children tend to develop pauci-bacillary TB and are rarely sputum smear positive. M. tuberculosis culture is more sensitive than smear microscopy, but it remains an insensitive test (culture yields of $30-40 \%$ are generally reported in children with probable TB) and is severely hampered by the difficulty of specimen collection, cost and a time delay of 2-6 wk. Immunologic approaches have not been very fruitful. The tuberculin skin test (TST), as well as novel interferon-gamma release assays, fail to make the all important distinction between latent infection and active disease (2).

Symptom-based approaches seemed to offer a practical solution in resource-limited settings but elicited a lot of skepticism, as previous approaches were poorly validated (3). A prevalence survey demonstrated that poorly defined symptoms, such as chronic cough, are commonly reported in children without TB, which limits its discriminatory power (4). However, the use of well-defined symptoms offered greatly improved diagnostic accuracy (5). In a prospective community-based study conducted in Cape Town, South Africa the combined presence of three symptoms at presentation; a persistent, nonremitting cough of $>2$ wk duration, documented failure to thrive in the preceding 3 mo and reduced playful- ness/fatigue diagnosed pulmonary TB with a good degree of accuracy in HIV-uninfected children (6). In those children who failed to meet these strict symptom criteria, simple clinical follow up proved very useful. Unfortunately, symptom-based diagnosis was far less accurate in immunocompromised HIV-infected children, with both reduced sensitivity due to acute disease progression, and reduced specificity due to chronic symptomatology associated with HIV and/or other opportunistic infections (6).

The most common extra-pulmonary disease manifestation of child TB is cervical adenitis. In this setting a cervical mass of $>2 \times 2 \mathrm{~cm}$, persisting for $>4 \mathrm{wk}$ and without a visible local cause (e.g., an infected Tinea capitis lesion on the scalp), was nearly pathognomonic for $\mathrm{TB}$, as proven on fine needle aspiration biopsy (7). Symptom-based approaches may also be used to exclude TB disease in TB contacts, thereby improving access to isoniazid preventive therapy in settings where TST and chest radiography are not readily available (8).

In the absence of novel diagnostic advances, refined symptom-based approaches may be of great value in resourcelimited settings, especially in HIV-uninfected children, who represent the majority of child TB cases even in HIV-endemic countries (9). - Ben J. Marais

\section{REFERENCES}

1. Marais BJ, Obihara CC, Warren RM, Schaaf HS, Gie RP, Donald PR 2005 The burden of childhood tuberculosis: a public health perspective. Int J Tuberc Lung Dis 9:1305-1313

2. Marais BJ, Pai M 2007N New approaches and emerging technologies in the diagnosis of childhood tuberculosis. Paediatr Respir Rev 8:124-133

3. Hesseling AC, Schaaf HS, Gie RP, Starke JR, Beyers N 2002 A critical review of diagnostic approaches used in the diagnosis of childhood tuberculosis. Int J Lung Dis 6:1038-1045

4. Marais BJ, Obihara CC, Gie RP, Schaaf HS, Hesseling AC, Lombard C, Enarson D, Bateman E, Beyers N 2005 The prevalence of symptoms associated with pulmonary tuberculosis in randomly selected children from a high-burden community. Arch Dis Child 90:1166-1170

5. Marais BJ, Gie RP, Obihara CC, Hesseling AC, Schaaf HS, Beyers N 2005 Welldefined symptoms are of value in the diagnosis of childhood pulmonary tuberculosis. Arch Dis Child 90:1162-1165

6. Marais BJ, Gie RP, Schaaf HS, Hesseling AC, Lombard C., Enarson DA, Beyers N. 2006 A refined symptom-based approach to diagnose pulmonary tuberculosis in children. Pediatrics e1350.

7. Marais BJ, Wright CA, Schaaf HS, Gie RP, Hesseling AC, Enarson DA, Beyers N 2006 Etiology of persistent cervical adenopathy in children: a prospective community-based study in an area with a high incidence of tuberculosis. Pediatr Inf Dis J 25:142-146

8. Kruk A, Gie RP, Schaaf HS, Marais BJ. 2007 Symptom-based screening of child TB contacts: improved feasibility in resource-limited settings. Pediatrics, In press

9. Marais BJ, Cotton M, Graham S, Beyers N 2007 Diagnosis and management challenges of childhood TB in the era of HIV. Jnl Infect Dis 196:S76-S85 